\title{
Sphingosine-1-phosphate (S1P) signaling regulates the production of intestinal IgA and its potential role in the pathogenesis of canine inflammatory bowel disease
}

\author{
Maho NAKAZAWA ${ }^{1)}$, Shingo MAEDA ${ }^{1) *}$, Nozomu YOKOYAMA ${ }^{2)}$, \\ Taisuke NAKAGAWA ${ }^{2)}$, Tomohiro YONEZAWA ${ }^{1)}$, Koichi OHNO ${ }^{2)}$ and \\ Naoaki MATSUKI ${ }^{1)}$ \\ ${ }^{1)}$ Department of Veterinary Clinical Pathobiology, Graduate School of Agricultural and Life Sciences, \\ The University of Tokyo, 1-1-1 Yayoi, Bunkyo-ku, Tokyo 113-8657, Japan \\ 2) Department of Veterinary Internal Medicine, Graduate School of Agricultural and Life Sciences, The University \\ of Tokyo, 1-1-1 Yayoi, Bunkyo-ku, Tokyo 113-8657, Japan
}

\section{J. Vet. Med. Sci.}

81(9): 1249-1258, 2019

doi: 10.1292/jvms.19-0016

Received: 10 January 2019

Accepted: 9 July 2019

Advanced Epub: 25 July 2019

\begin{abstract}
Inflammatory bowel disease (IBD) is a common gastrointestinal disease in dogs. Decreased production of intestinal immunoglobulin $A(\lg A)$ has been suggested as a possible pathogenesis in a subset of canine IBD; however, the underlying cause remains unclear. Sphingosine-1-phosphate (S1P) is a lipid mediator that regulates intestinal IgA production by controlling lymphocyte trafficking in mice. The objectives of this study were to clarify the role of S1P in IgA production in dogs and to evaluate the expression of S1P-related molecules in dogs with IBD. First, an S1P receptor antagonist was administrated to five healthy dogs. The S1P receptor antagonist significantly decreased the IgA concentration in sera and feces but did not affect the IgG concentration. Moreover, the immunoreactivity of intestinal IgA was significantly decreased by S1P signal blockade. These results indicate that S1P signaling specifically regulates the intestinal IgA production in dogs. Subsequently, the intestinal S1P concentration and the expression of S1P-related molecules were measured in dogs with IBD and healthy dogs. The intestinal concentration of S1P was significantly lower in dogs with IBD than in healthy dogs. In addition, the gene expression levels of S1P receptor (S1P1) and S1P synthase (SK1) were significantly lower in dogs with IBD than in healthy dogs. Taken together, these observations suggest that decreased S1P production, likely caused by a lower expression of S1P synthetase, leads to attenuation of S1P/S1P1 signaling pathway and the production of intestinal IgA in dogs with IBD.
\end{abstract}

KEY WORDS: canine, chronic enteropathy, immunoglobulin, lipid mediator, mucosal immunity

Inflammatory bowel disease (IBD) in dogs is a heterogeneous group of chronic enteropathies characterized by continuous or recurrent gastrointestinal clinical signs such as vomiting, diarrhea, and weight loss [17]. In the intestine of dogs with IBD, overexpression of inflammatory cytokines and an increased number of inflammatory cells, including $\mathrm{CD}^{+} \mathrm{T}_{\text {cells, }} \mathrm{IgG}^{+}$cells, macrophages, and neutrophils, are observed [9, 18, 24]. These observations indicate the excessive immune reaction during canine IBD. Although the etiology and pathogenesis of chronic enteritis remain unclear, it is thought to develop due to an alteration of the bacterial flora, disruption of the mucosal barrier, and dysregulation of the mucosal immune responses [10]. Previous microbiome studies have reported the alteration of intestinal bacterial flora in dogs with IBD, compared with healthy dogs [16, 28]. In addition, the expression levels of Toll-like receptors (TLRs), which recognize bacterial components and trigger the inflammatory response, are upregulated in dogs with IBD [4, 27]. These results suggest that an imbalance of bacterial flora and uncontrolled TLR activation lead to the development of IBD lesions through overexpression of inflammatory cytokines. However, the intestinal mucosal immunity usually prevents bacterial invasion and aberrant TLR activation, implying that abnormalities in mucosal immune protection may play a central role in the development of canine IBD.

A key strategy of mucosal immunity is the production of immunoglobulin A (IgA). IgA is the most abundant antibody isotype in the intestinal mucosa, which sets up the barriers that inhibit excessive immune response to harmless antigens [5]. For example, IgA

*Correspondence to: Maeda, S.: amaeda@mail.ecc.u-tokyo.ac.jp (Supplementary material: refer to PMC https://www.ncbi.nlm.nih.gov/pmc/journals/2350/)

(O2019 The Japanese Society of Veterinary Science 
neutralizes pathogens and exotoxins, and it prevents commensal bacteria from adhering to the intestinal epithelium [21]. Unlike IgG or IgM, IgA functions without activating complementary cascades, thereby, inhibiting excessive or chronic inflammation in the gastrointestinal tract [14]. A previous study demonstrated that, compared with healthy dogs, the fecal and intestinal IgA concentration is decreased in dogs with IBD [23]. In addition, $\operatorname{IgA}^{+} \mathrm{CD} 21^{+} \mathrm{B}$ cells in the peripheral blood and $\operatorname{Ig} \mathrm{A}^{+}$cells in the intestinal lamina propria are also decreased in dogs with IBD. These results indicate that decrease in intestinal IgA production is a possible pathogenesis for a subset of dogs with canine IBD. Intestinal IgA production comprises three steps: (1) class switching of naive B cells into $\operatorname{IgA}^{+} \mathrm{B}$ cells after antigen presentation in the Peyer's patches (organized lymphoid tissues in the gut), (2) migration of $\operatorname{IgA}^{+} \mathrm{B}$ cells into the lamina propria of intestine via lymphatic and blood vessels, and (3) differentiation of $\operatorname{Ig} \mathrm{A}^{+} \mathrm{B}$ cells into $\operatorname{IgA}^{+}$plasma cells and production of IgA. In dogs with IBD, an abnormality is expected to occur somewhere in this three-step process.

Sphingosine-1-phosphate (S1P) is a bioactive lipid mediator that has been identified as an important molecule in the regulation of lymphocyte egress from the thymus and secondary lymph nodes [2, 7]. S1P is present abundantly in the blood and lymph, produced mainly by platelets, erythrocytes, and endothelial cells, and production of S1P is catalyzed by sphingosine kinase 1 and 2 (SK1 and SK2) [29, 32]. S1P is degraded by S1P lyase [34]. So far, five types of S1P receptors (S1P1-S1P5) have been identified $[3,32]$. Of these, S1P1 is preferentially expressed by lymphocytes, and it senses S1P in the lymph and blood to regulate the lymphocyte egress from organized lymphoid tissue [26]. Previous studies on murine models have shown that S1P/S1P1 axis plays an important role in intestinal IgA production by accelerating the egress of mature B cells from Peyer's patch and their immigration from the blood circulation into the peritoneal cavity and then intestinal lamina propria $[11,20]$. Hence, clarification of the role of $\mathrm{S} 1 \mathrm{P}$ in lymphocyte immigration and immunoglobulin production would help elucidate the pathophysiological mechanism of canine IBD.

This study first sought to investigate the effect of S1P signal blockade on canine lymphocyte trafficking and intestinal IgA production using FTY720, an inhibitor of S1P receptors $[6,13]$. FTY720 blocks S1P signaling in canine cells by internalization of $\mathrm{S} 1 \mathrm{P}$ receptors [33]. In addition, the expression levels of intestinal S1P and S1P-related molecules were examined in dogs with IBD and healthy control dogs.

\section{MATERIALS AND METHODS}

\section{FTY720 administration to healthy dogs and sample collection}

Five healthy Beagle dogs were used in this experiment. All of them were neutered males, and their median age was 49 months (range: $48-50$ months). The median body weight was $10.16 \mathrm{~kg}$ (range: $9.68-10.7 \mathrm{~kg}$ ). The dogs were healthy with no clinical signs of gastrointestinal disease, and they had received no medication. No abnormalities were observed in the complete blood count, serum biochemistry, urinalysis, or the parasitic and bacterial PCR analyzes of feces (Canine Diarrhea RealPCR ${ }^{\mathrm{TM}}$ Panel, IDEXX Laboratories, Maine, IL, U.S.A.).

The experimental outline is shown in Fig. 1. FTY720 (Gilenya ${ }^{\circledR}$, Novartis Pharma, Basel, Switzerland) was given orally to the dogs at a dose of $0.01 \mathrm{mg} / \mathrm{kg}$ once a day, for 2 weeks. During the medication period, clinical symptoms (general condition, appetite, vomiting, diarrhea, and drinking volume) were monitored every day and physical examinations (body temperature, heart rate, respiratory rate, and body weight) were performed every week. Blood (whole blood, plasma, and serum) and feces were collected pre- and post-administration and two and four weeks after administration. Duodenal samples were obtained by endoscopic biopsy under general anesthesia pre- and post-administration (Fig. 1). The use of dogs in this experiment was approved by the Animal Care Committee of The University of Tokyo (approval no. P17-108).

\section{Blood examination}

Whole blood collected in an EDTA tube was examined using a hematology analyzer (ProCyte Dx, IDEXX Laboratories). Blood smear specimens were prepared and stained using Wright-Giemsa stain. White blood cells on the smear specimens were counted under a microscope and categorized into neutrophils, eosinophils, basophils, lymphocytes, and monocytes. The result was expressed as the number of cells per microliter.

Plasma was collected to measure the blood urea nitrogen (BUN), creatinine, total protein, albumin, canine C-reactive protein (CRP), sodium, potassium, and chloride concentrations, as well as alanine aminotransferase (ALT) and alkaline phosphatase (ALP) enzyme activity, using a chemical analyzer (DRI-CHEM 7000 Z, FUJIFILM, Tokyo, Japan).

\section{$\operatorname{Ig} A$ and $\operatorname{IgG}$ ELISA}

$\operatorname{IgA}$ and $\operatorname{IgG}$ concentrations in the feces and sera were determined using commercial ELISA kits specific for canine IgA (Dog IgA ELISA Quantification Set, Bethyl Laboratories, Montgomery, TX, U.S.A.) and IgG (Dog IgG ELISA Quantification Set, Bethyl Laboratories). The fecal samples were centrifuged in a dilution buffer (50 mM Tris containing $0.14 \mathrm{M} \mathrm{NaCl}$ and $0.05 \%$ Tween 20) and coarse particles were removed from fecal supernatants by filtration using a $0.8-\mu \mathrm{m}$ syringe filter. Absorbance was recorded at $450 \mathrm{~nm}$ using a microplate reader (Bio Rad Laboratories, Hercules, CA, U.S.A.), and the results were analyzed using Microplate Manager software version 5.2.1 (Bio Rad Laboratories). All the samples were tested in duplicate, and the mean optical density (OD) was calculated. Total protein concentrations of the fecal samples were determined by the Lowry method (DC Protein Assay, Bio Rad Laboratories). Fecal IgA and IgG concentrations were normalized to micrograms per milligram of total protein. Serum IgA and IgG concentrations were expressed in milligrams per deciliter. 
Days

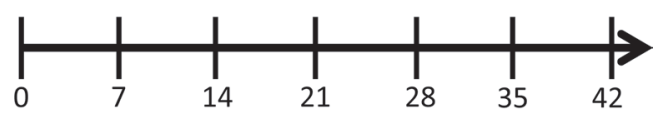

Monitoring of clinical signs
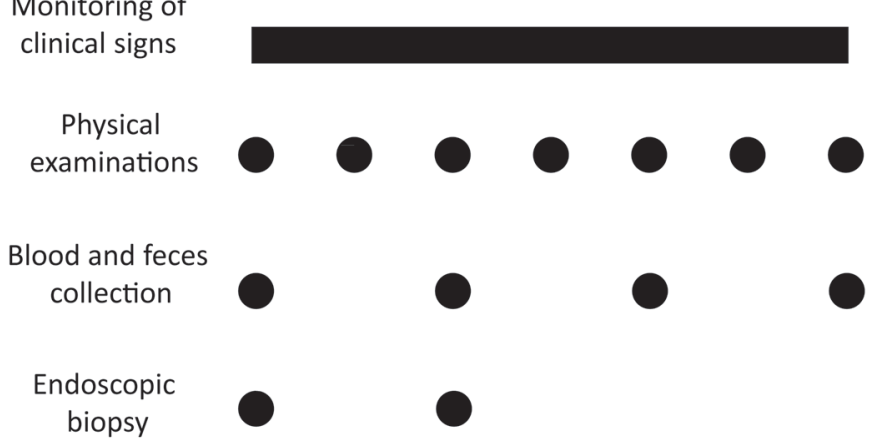

Fig. 1. Experimental outline of FTY720 administration and sample collection.

\section{Immunohistochemistry}

Immunohistochemistry (IHC) was conducted using paraffin-embedded sections (4- $\mu \mathrm{m}$ thick) of duodenal samples obtained by endoscopic biopsy. Heat-induced antigen retrieval was performed by autoclaving for $10 \mathrm{~min}$ at $121^{\circ} \mathrm{C}$ in $10 \mathrm{mM}$ sodium citrate buffer (pH 6.0). The endogenous peroxidase was blocked using a blocking solution (REAL Peroxidase-Blocking Solution, DAKO, Glostrup, Denmark) for $10 \mathrm{~min}$ at room temperature. The sections were blocked with 5\% skimmed milk in TBS for 60 min at room temperature, and then incubated overnight at $4{ }^{\circ} \mathrm{C}$ with antibodies. Primary antibodies, anti-human CD3 antibody (Envision FLEX-CD3, DAKO; ready to use), anti-human CD20 antibody (Envision FLEX-CD20cy (L26), DAKO; ready to use), anti-dog IgA antibody (Goat anti-Dog IgA Antibody, Bethyl laboratories; 1:1,000 dilution), and anti-dog IgG antibody (Rabbit anti-Dog IgG Antibody, Bethyl laboratories; 1:5,000 dilution) were used. After washing with TBS, the samples were incubated for 40 min at $37^{\circ} \mathrm{C}$ with HRP-labeled antirabbit IgG (EnVision ${ }^{+}$System ${ }^{-}$HRP Labeled Polymer Anti-Rabbit, DAKO; ready to use) for CD3 and IgG, and with HRP-labeled anti-mouse IgG (EnVision ${ }^{+}$System $^{-}$HRP Labeled Polymer Anti-Mouse, DAKO; ready to use) for CD20. For IgA, the samples were incubated with a biotinylated anti-goat IgG antibody (Biotinylated Anti-Goat IgG (H+L), Vector Laboratories, Burlingame, CA, U.S.A.; 1:400 dilution) for $40 \mathrm{~min}$ at $37^{\circ} \mathrm{C}$, and then incubated with HRP-labeled streptavidin (HRP-labeled streptavidin, DAKO; 1:500 dilution) for $40 \mathrm{~min}$ at room temperature. The reaction products were visualized with 3,3'-diaminobenzidine.

Ten regions were chosen at random for each slide using a light microscope with a $20 \times$ objective lens and a $10 \times$ eyepiece lens. Digitized images were transferred to a computer to measure the size of each region using a software (ImageJ, National Institute of Mental Health, Bethesda, MD, U.S.A.). Lamina propria CD3 ${ }^{+}$cells, CD20 cells, $\mathrm{IgA}^{+}$cells, and $\mathrm{IgG}^{+}$cells in each area were counted. The results were expressed as positive cells per $10,000 \mu \mathrm{m}^{2}$.

For IgA and IgG evaluation, the immunoreactivity score of each slide was also determined. The score (0-3) of each biopsy specimen was determined as follows: score 0: no cell was stained in the area, score 1: cells were weakly stained with a vague staining of cell membrane, score 2: cells were stained with clear staining of cell membrane, score 3: cells were strongly stained with a clear staining of cell membrane. Representative pictures for scores 1-3 are shown in Supplementary Fig. 1. The score for each slide was determined as the average score of the specimens on the slide.

\section{Gene expression of S1P receptors in canine lymphocytes, monocytes, and intestine in healthy dogs}

Peripheral blood mononuclear cells (PBMCs) were collected from fresh EDTA blood samples of healthy Beagles using a density gradient cell separation medium (HISTOPAQUE-1077, Sigma-Aldrich, St. Louis, MO, U.S.A.). PBMCs were incubated overnight at $37^{\circ} \mathrm{C}$ in a culture medium (RPMI-1640, Wako, Tokyo, Japan) to isolate the lymphocytes as floating cells. The adhered cells were collected as monocytes [12]. Total mRNA was extracted from lymphocytes and monocytes using a commercially available kit (RNAspin Mini RNA Isolation Kit; GE Healthcare, Buckinghamshire, England), according to the manufacturer's instructions. The DNA was amplified by reverse transcription PCR using a commercial kit (KOD FX Neo, TOYOBO, Osaka, Japan). Expression of S1P1-S1P5 and GAPDH was detected using an automatic electrophoresis system (QIAxceL Advanced System, QIAGEN, Hulsterweg, Netherlands). In addition, $\mathrm{CD}^{+} \mathrm{T}$ cells and $\mathrm{CD} 21^{+} \mathrm{B}$ cells were sorted from the canine PBMCs using a cell sorter (BD FACSAria ${ }^{\mathrm{TM}}$ cell sorter, Becton, Dickinson and Co., Franklin Lakes, NJ, U.S.A.). CD21 is used as B cell maker in canine peripheral blood. Intestinal expression of S1P1-S1P4 and GAPDH was also examined in duodenal samples obtained using endoscopic biopsy from healthy dogs. The primer pair sequences used in this experiment are shown in Supplementary Table 1. 


\section{Study population}

The study included dogs diagnosed with IBD that underwent endoscopic examination in the Veterinary Medical Center of the University of Tokyo (VMC-UT). Informed consent was obtained from all owners, and the study protocols were approved by the Animal Care Committee of the VMC-UT. The case selection criteria for IBD have been previously described [22]. Diagnosis of IBD was based on: (1) exclusion of other identifiable causes of chronic gastrointestinal signs by complete blood count, serum biochemistry, urinalysis, parasitic and bacterial PCR analyzes of fecal samples, and abdominal ultrasonography, (2) histopathologic evidence of lymphocytic-plasmacytic enteritis, (3) the absence of clonal gene rearrangement of $\operatorname{IgH}$ or $T C R \gamma$ in the PCR for antigen receptor gene rearrangement (PARR), and (4) exclusion of food- and antibiotic-responsive enteritis. The cases with a discrepancy between histopathology and PARR results were excluded. In addition, dogs that had been treated with corticosteroids during the 2 weeks before the study were also excluded.

For S1P ELISA, 25 dogs with IBD were included in the study. The median age of these dogs was 88 months (range: 38-139 months); seven of the dogs were female ( 3 intact and 4 neutered) and the remaining 18 dogs were male (11 intact and 7 neutered). The median body weight was $6.06 \mathrm{~kg}$ (range: $2.12-9.5 \mathrm{~kg}$ ). The following breeds with more than two individuals were included: French Bulldog $(n=4)$, Boston Terrier $(n=3)$, Yorkshire Terrier $(n=3)$, Miniature Dachshund $(n=3)$, Chihuahua $(n=2)$, and Cavalier King Charles Spaniel $(n=2)$. The remaining eight dogs were of other breeds. Sixteen beagles were included as healthy controls. The median age of these dogs was 62 months (range: 49-110 months); six of the dogs were female (1 intact and 5 neutered) and the other ten dogs were male (4 intact and 6 neutered). The median body weight was $10.62 \mathrm{~kg}$ (range: 9.98-12.1 kg). The use of dogs in this study was approved by the Animal Care Committee of the University of Tokyo (approval no. P11-530 and P17-108). A summary of the characteristics of dogs used in this experiment is shown in Supplementary Table 2.

For quantitative real-time PCR, 42 dogs diagnosed with IBD were included in this study. The median age of these dogs was 89 months (range: $22-160$ months); 17 of the dogs were female ( 7 intact and 10 neutered) and the remaining 25 dogs were male (17 intact and 8 neutered). The median body weight was $5.55 \mathrm{~kg}$ (range: $2.2-32.4 \mathrm{~kg}$ ). The following breeds with more than two individuals were included: Miniature Dachshund $(n=5)$, Yorkshire Terrier $(n=5)$, Pembroke Welsh Corgi $(n=3)$, Chihuahua $(n=3)$, Pug ( $n=3)$, Cavalier King Charles Spaniel $(n=3)$, French Bulldog $(n=3)$, Boston terrier $(n=2)$, Shiba Inu $(n=2)$, Jack Russel Terrier $(n=2)$, and Maltese Terrier $(n=2)$. The remaining nine dogs were of other breeds. Nine beagles were included as healthy controls and duodenal samples were obtained by endoscopic biopsy. The median age of these dogs was 90 months (range: 49-110 months); four of the dogs were female ( 1 intact and 3 neutered) and the remaining five dogs were male (all intact). The median body weight was $11 \mathrm{~kg}$ (range: $10.4-15 \mathrm{~kg}$ ). Characteristics of the dogs used for quantitative real-time PCR are summarized in Supplementary Table 3.

\section{SIP ELISA}

Duodenal S1P concentrations were determined using a commercial ELISA kit (Abbexa, Cambridge, U.K.). Duodenal samples obtained by endoscopy were snap-frozen in liquid nitrogen and stored at $-80^{\circ} \mathrm{C}$ for later use. The samples were weighed and then rinsed with ice-cold PBS to remove the excessive blood. Next, they were homogenized in $1 \mathrm{~m} l$ of PBS and centrifuged, and the supernatant was used for the ELISA. Absorbance was recorded at $450 \mathrm{~nm}$ using a microplate reader (Bio Rad Laboratories) and the results were analyzed using Microplate Manager software, version 5.2.1 (Bio Rad Laboratories). All the samples were tested in duplicate, and the mean optical density (OD) was calculated. S1P concentration was expressed as nanograms per $100 \mu \mathrm{g}$ of tissue.

\section{Quantitative real-time PCR}

Total RNA was extracted from the duodenal samples as previously described. Genomic DNA was removed from the samples using the TURBO DNA-free Kit (Applied Biosystems, Waltham, MA, U.S.A.), and the samples were stored at $-80^{\circ} \mathrm{C}$ until later use. Expression levels of S1P1, S1P synthase 1 and 2 (SK1 and SK2), and S1P lyase (SPGL1) mRNA were quantified by two-step real-time RT-PCR (StepOnePlus; Applied Biosystems) using a commercial kit (PrimeScript RT Reagent Kit and SYBR PrimeScript RT-PCR Kit II, Takara Bio, Kusatsu, Japan), according to the manufacturer's instructions. RPL32 and GAPDH were chosen as the reference genes in this study. The primer pair sequences used in this study are shown in Supplementary Table 3. All the samples were examined in duplicate and the mean $\Delta \mathrm{Ct}$ value was calculated. The relative expression of the target gene is reported as the $n$-fold difference relative to reference gene expression, by subtracting $\mathrm{Ct}$ values of the reference gene from the $\mathrm{Ct}$ values of the target $(\Delta \mathrm{Ct})$. Each PCR reaction included a no-template control with sterile distilled water instead of the cDNA template, to test for contamination of assay reagents or primer pairs. The melting curve analysis did not show a misprinting in any of the reactions.

\section{Statistical analysis}

Statistical analyzes were performed using a software (Prism version 5.0b, GraphPad Software, La Jolla, CA, U.S.A.). Friedman test followed by Dunn's test was used to detect the change in the number of lymphocytes, the concentration of serum, and fecal IgA and IgG. Wilcoxon's test was used to compare the number of $\mathrm{CD}^{+}, \mathrm{CD}_{2} 0^{+}, \mathrm{IgA}^{+}$, and $\mathrm{IgG}^{+}$cells, and the score of immunohistochemistry for IgA and IgG. In addition, Mann-Whitney $U$-test was used to compare the gene expression levels and S1P concentrations. Results were considered statistically significant at $P<0.05$. 


\section{RESULTS}

\section{Effect of FTY720 on the general condition and blood examination}

No dog showed a decrease in body weight or gastrointestinal symptoms, including vomiting and diarrhea, during or after the administration of FTY720. At the end of the medication period, three out of the five dogs showed a slight increase in CRP $(0.9,1.0$, $1.6 \mathrm{mg} / \mathrm{d} l$; reference range $<0.7)$, three dogs showed a slight increase in sodium $(154,155,162 \mathrm{mEq} / l$; reference range $141-152$ $\mathrm{mEq} / l)$, two dogs showed a slight increase in chloride $(123,125 \mathrm{mEq} / l$; reference range $102-117 \mathrm{mEq} / l)$, and two dogs showed a moderate elevation in ALP enzyme activity $(324,353 \mathrm{U} / l$; reference range 47-254 U/l). All these abnormalities recovered 2 weeks after the end of the discontinuation. No dog showed an abnormality in BUN, creatinine, total protein, albumin, potassium concentration, and ALT enzyme activity.

During the complete blood count examination, red blood cells and platelets were found to be stable during the observation period. In contrast, white blood cells were significantly decreased after the administration of FTY720 ( $P=0.0013$, Fig. 2A). Of the white blood cells, only lymphocytes were significantly decreased after the administration of FTY720, compared with the pre-treatment levels $(P<0.0001$, Fig. 2B). The number of white blood cells as well as lymphocytes recovered gradually after discontinuation. There was no significant difference in the number of neutrophils, basophils, eosinophils, and monocytes, between pre- and post-FTY720 administration data.

\section{Effect of FTY720 on the fecal and serum immunoglobulin concentration}

FTY720 decreased the fecal and serum IgA concentration during the observation period (Fig. 3). While the fecal IgA concentration did not change during the medication period, it was decreased significantly 2 weeks after the discontinuation, compared with the baseline $(P<0.001$ Fig. $3 \mathrm{~A})$. The serum IgA concentration started to decrease at the end of the medication period, and decreased significantly 2 weeks after discontinuation $(P<0.001$, Fig. 3 C). FTY720 treatment did not affect the IgG concentration during the observation period (Fig. 3B and 3D).

\section{Effect of FTY720 on the intestinal lymphocytes}

Representative images of the IHC results before and after the FTY720 treatment are shown in Fig. 4. There was no significant difference in the number of $\mathrm{CD}^{+} \mathrm{T}$ cells between pre- and post-treatment data (Fig. 5A). On the other hand, FTY720 treatment significantly decreased the number of $\mathrm{CD} 20^{+} \mathrm{B}$ cells $(P=0.00257$; Fig. $5 \mathrm{~B})$.

Subsequently, we examined which type of B cells, $\mathrm{IgG}^{+}$or $\mathrm{IgA}^{+}$cells, were decreased by FTY720 treatment. There was no significant difference in the number of $\mathrm{IgA}^{+}$and $\mathrm{IgG}^{+}$cells between pre- and post-FTY720 administration data (Fig. 5C and 5D), although $\mathrm{IgA}^{+}$cells tended to decrease after the FTY720 treatment. Since IgA immunoreactivity was likely to decrease after the administration of FTY720 (Fig. 4), the immunoreactivity scores of IgA and IgG were evaluated. The score of IgA immunoreactivity after the administration was significantly lower than that of the baseline $(P=0.0168$; Fig. 5E), whereas there was no significant difference in the IgG immunoreactivity score (Fig. 5F).

\section{Gene expression of SIP receptors in canine lymphocytes, monocytes, and intestine}

Gene expression levels of five types of S1P receptors (S1P1-S1P5) in the canine peripheral blood lymphocytes and monocytes were evaluated to examine which cells were influenced by FTY720 treatment. The representative images of electrophoresis are shown in Fig. 6. Canine lymphocytes and monocytes expressed S1P1, S1P2, and S1P4, but not S1P3 and S1P5. Of the lymphocytes, $\mathrm{CD} 21^{+} \mathrm{B}$ cells strongly expressed $S 1 P 1$ and $S 1 P 4$ relative to the $\mathrm{CD}^{+} \mathrm{T}$ cells (Fig. 6). These results suggest that

A

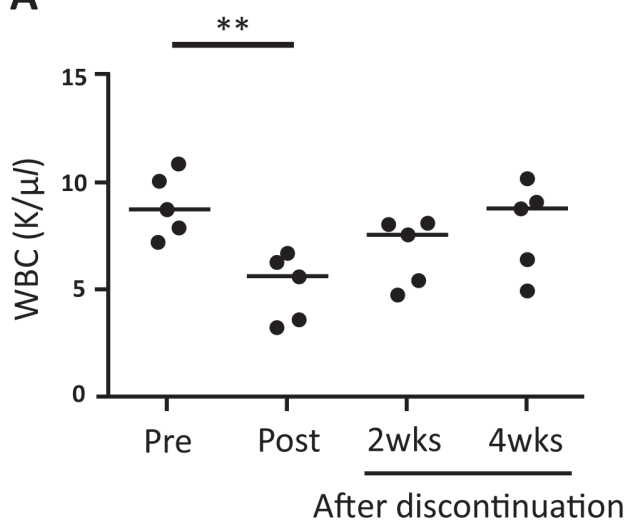

B

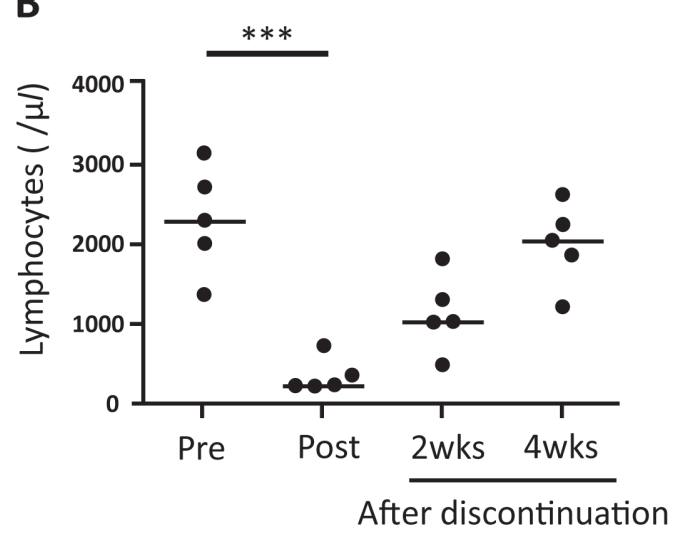

Fig. 2. The number of white blood cells (WBC, A) and lymphocytes (B) during and after the medication period. Horizontal lines indicate median. Pre, before the administration. Post, after the administration. 2 wks, 2 weeks after discontinuation. 4 wks, 4 weeks after discontinuation. $* * P<0.01$. *** $P<0.001$. 
A

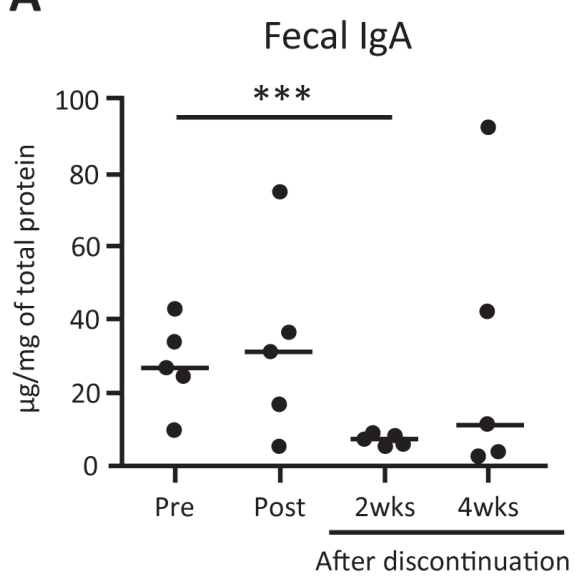

C

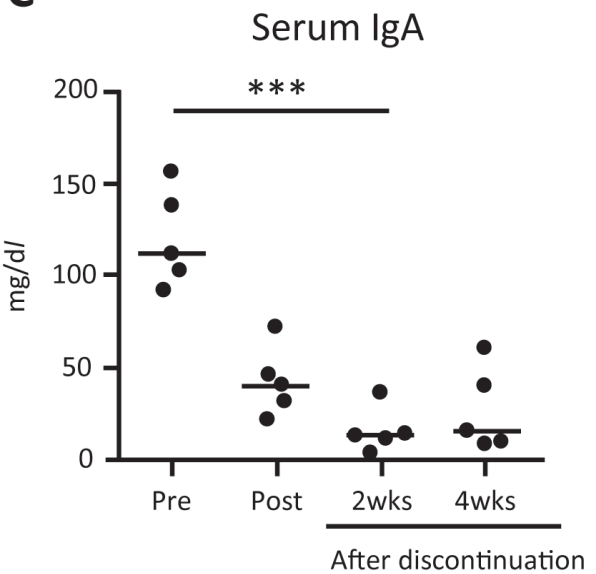

B

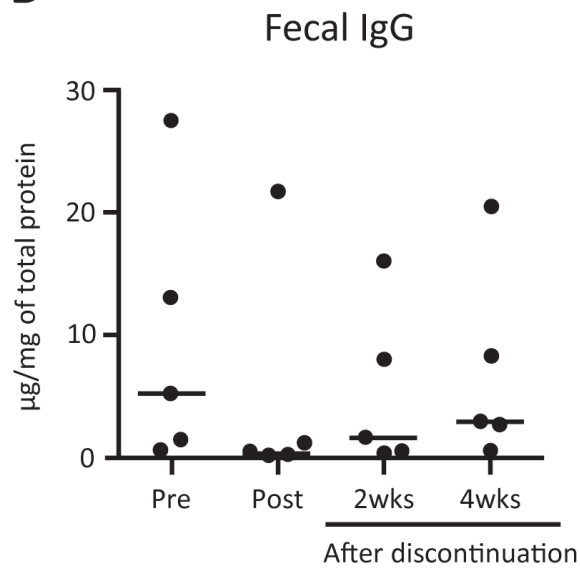

D

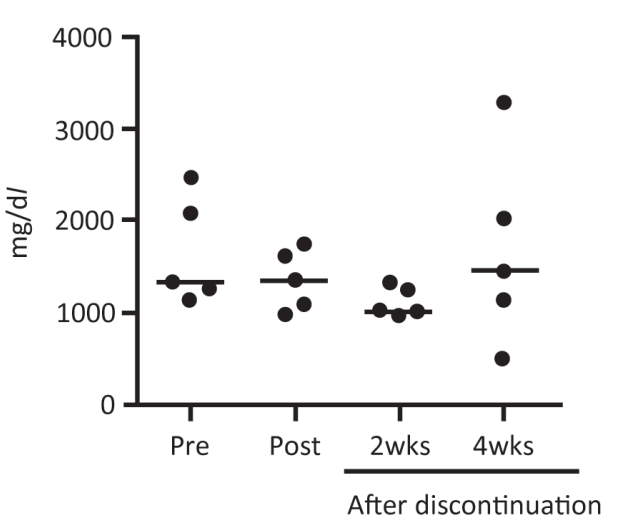

Fig. 3. $\operatorname{IgA}($ left $)$ and $\operatorname{IgG}$ (right) concentration in feces (A and B) and serum (C and D). Horizontal lines indicate median. Pre, before the administration. Post, after the administration. 2 wks, 2 weeks after discontinuation. 4 wks, 4 weeks after discontinuation. $* * * P<0.001$. N.S., not significant.

the effect of FTY720 was induced through blockade of S1P1 and/or S1P4. To examine the expression pattern of S1P receptors in canine intestines, conventional PCR was performed. Of the five S1P receptors, only S1P1 was detected in the canine intestine (Fig. $6)$.

\section{Intestinal S1P concentration in healthy dogs and dogs with IBD}

In healthy dogs, the duodenal S1P concentration varied widely among the individuals (Fig. 7A). In contrast, duodenal S1P concentration in all the cases with IBD was very low. The concentration of duodenal S1P in dogs with IBD was significantly lower than that in healthy dogs $(P=0.0002$; Fig. $7 \mathrm{~A})$.

\section{Intestinal gene expression of SIP1 in healthy dogs and dogs with IBD}

Of the five S1P receptors, only S1P1 was detected in the canine intestine. Thus, further expression analysis of intestinal S1P1 in dogs with IBD and healthy controls was conducted by quantitative real-time PCR. The intestinal expression levels of S1P1 in dogs with IBD were significantly lower than those in healthy dogs $(P=0.0012$, Fig. 7B).

\section{Intestinal gene expression of enzymes regulating S1P concentration in healthy dogs and dogs with IBD}

To examine the cause of decreased S1P concentration in dogs with IBD, we examined the gene expression of enzymes that regulate S1P concentration, sphingosine kinases (SK1 and SK2), and S1P lyase (SGPL1). The expression levels of SK1 were significantly decreased in dogs with IBD compared with healthy dogs $(P=0.0266$; Fig. $7 \mathrm{C})$. In contrast, there was no significant difference in $S K 2$ and $S G P L 1$ expression between the two groups. 


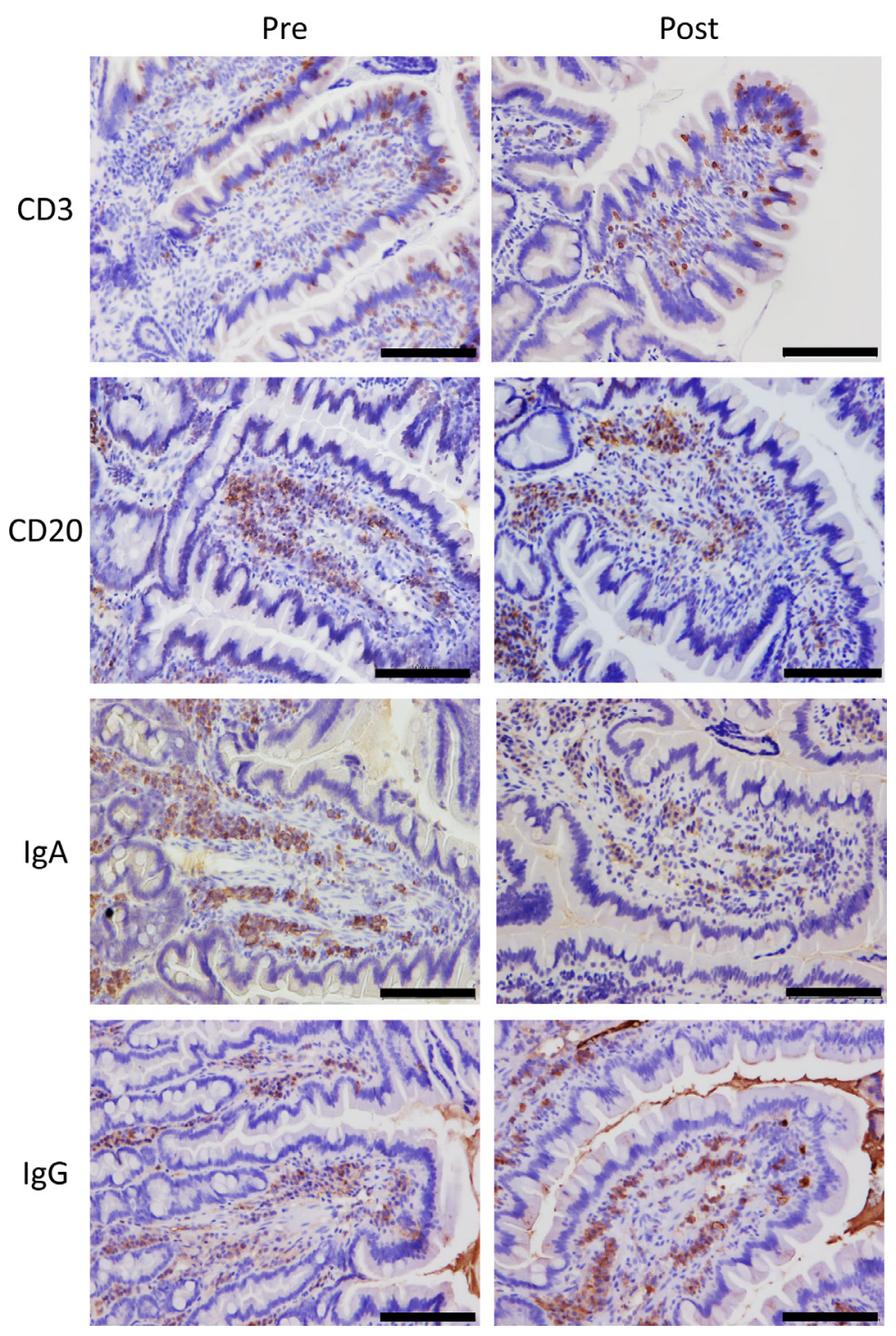

Fig. 4. Representative images of IHC for CD3, CD20, IgA, and IgG in duodenum before (left) and after (right) administration of FTY720. Bars $=100 \mu \mathrm{m}$.

\section{DISCUSSION}

In this study, we demonstrated that S1P signal blockade decreased the number of peripheral and intestinal lymphocytes, especially B cells, as well as IgA production. Moreover, we showed a decreased S1P concentration in the intestine of dogs with IBD. Together with the result of a previous study that IgA production is reduced in a subset of dogs with canine IBD [23], the present results suggest that S1P signaling is involved in the production of IgA, and its aberration leads to chronic enteritis and IBD through disruption of mucosal immunity.

Firstly, we investigated the effect of S1P signal blockade on intestinal lymphocyte trafficking and IgA production in healthy dogs using FTY720 [35]. The number of circulating lymphocytes was significantly decreased by S1P signal blockade. Previous studies on rats and mice have shown that FTY720 decreases the number of peripheral lymphocytes by inhibiting their emigration from thymus and secondary lymphoid organs $[6,15,38]$. In addition, the dose used in this study didn't induce apoptosis or cell death of canine lymphocytes [36]. As the other leukocytes were not affected by FTY720 treatment, the decreased number of total white blood cells likely reflects the decrease in the number of lymphocytes.

FTY720 blocks the S1P signaling by inhibiting all the S1P receptors, except S1P2 [6, 25, 35]. Our results showed that FTY720 specifically affected the number of circulating lymphocytes in dogs. In mice and humans, lymphocytes preferentially express S1P1 and S1P4 [2, 3, 30]. This result is consistent with canine lymphocytes. Therefore, S1P regulates peripheral lymphocyte circulation by binding of S1P1 or S1P4, or both. Interestingly, the expression pattern of S1P receptors in canine peripheral lymphocytes and monocytes was similar. Thus, the expression pattern of S1P receptors could not explain the specific decrease in circulating 
A

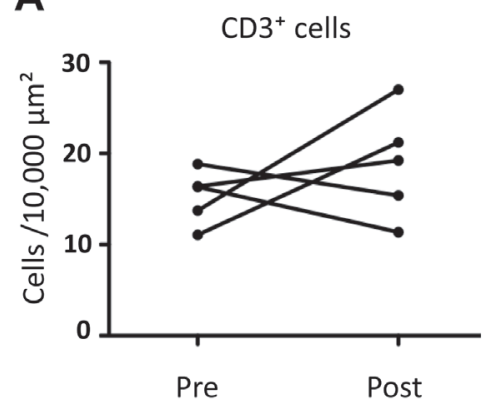

C

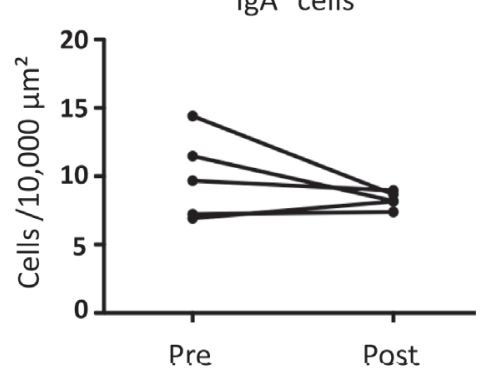

E

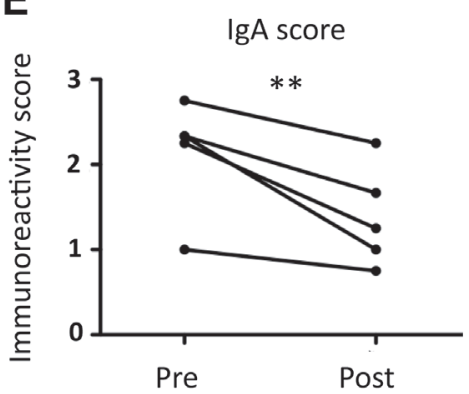

B

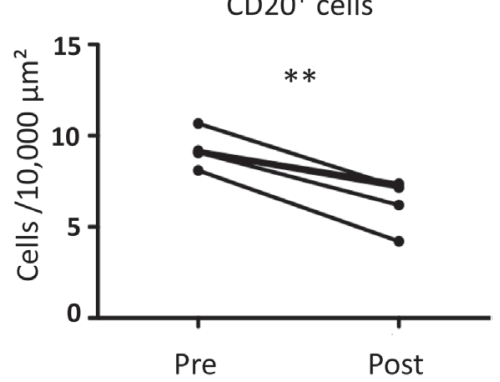

D

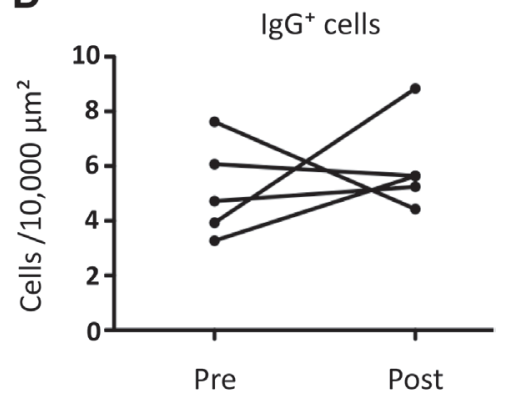

$\mathbf{F}$

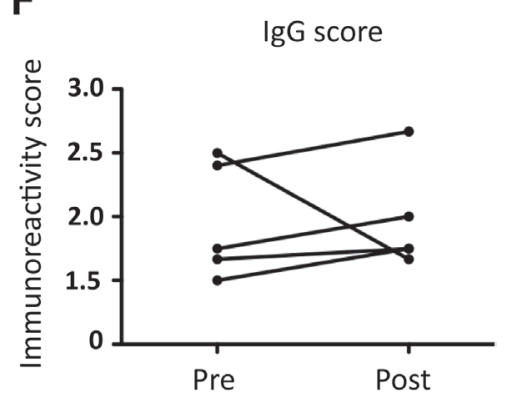

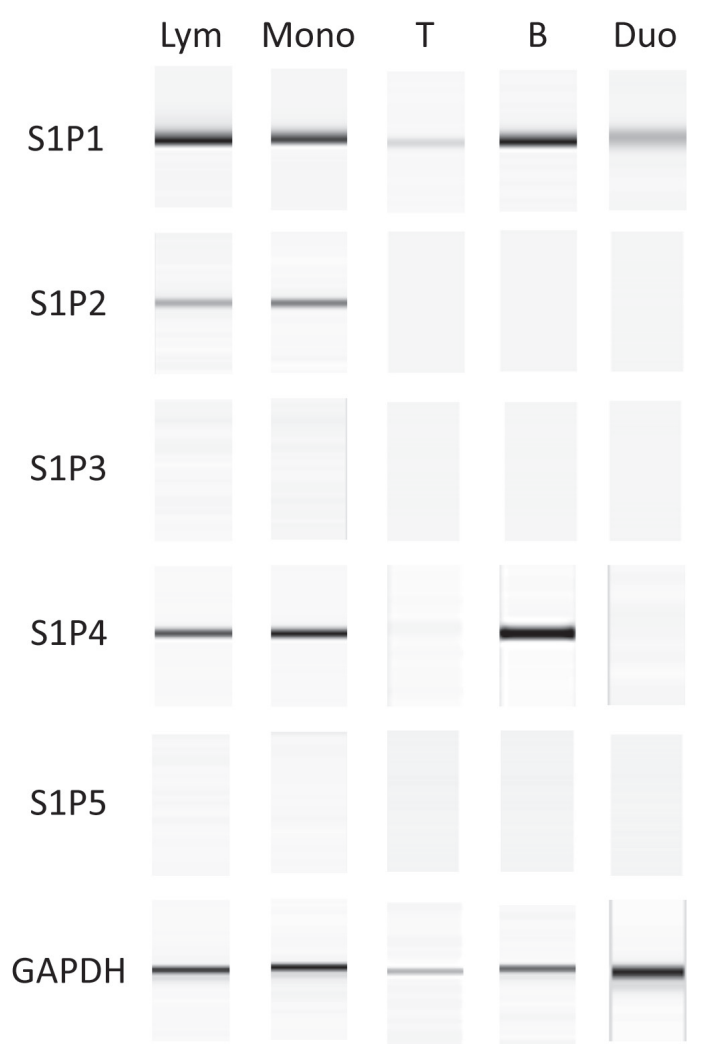

Fig. 6. Gene expression of S1P1-S1P5 in lymphocytes, monocytes, T cells, B cells, and duodenum of a representative healthy dog. Lym, lymphocytes. Mono, monocytes. T, T cells. B, B cells. Duo, duodenum.

Fig. 5. Change in the number of duodenal $\mathrm{CD}^{+}(\mathrm{A}), \mathrm{CD}_{2} 0^{+}(\mathrm{B}), \mathrm{IgA}^{+}(\mathrm{C})$, and $\mathrm{IgG}^{+}$(D) cells, and immunoreactivity scores of $\operatorname{IgA}(\mathrm{E})$ and $\operatorname{IgG}(\mathrm{F})$ before and after administration of FTY720. ${ }^{* *} P<0.01$. N.S., not significant.
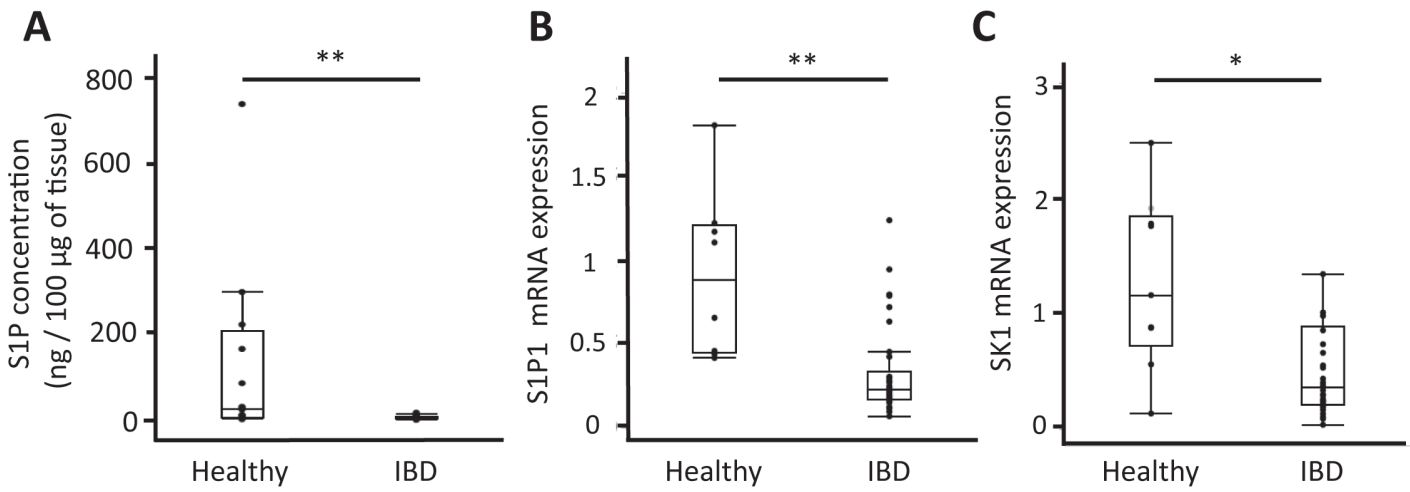

Fig. 7. The concentration of duodenal S1P (A) in healthy dogs $(n=16)$ and dogs with IBD $(n=25)$. The relative mRNA expression of $S 1 P 1$ (B) and $S K 1(\mathrm{C})$ in healthy dogs $(n=9)$ and dogs with IBD $(n=42)$. Horizontal line in each box indicates the median. Lower and upper boundaries indicate the 25 th and 75 th percentile, respectively. Whiskers represent the most extreme observations that were not outliners. $* * P<0.01$. $* P<0.05$. N.S, not significant. 
Further studies are needed to evaluate the effect of FTY720 on the chemokine signaling pathway in canine peripheral leukocytes.

Contrary to our expectation that FTY720 would decrease the number of both T and B cells in the canine intestine, FTY720 specifically decreased the number of duodenal CD20 $\mathrm{B}$ cells. The canine peripheral B cells showed a strong expression of S1P1 and S1P4 compared with the peripheral T cells, indicating that the B cell-specific effect of FTY720 may be due to the expression intensities of S1P receptors. As the canine duodenum expressed S1P1 but not S1P4, the S1P/S1P1 pathway may be important for the migration of $\mathrm{B}$ cells into intestines.

This study also showed that FTY720 treatment significantly decreased the fecal and serum IgA concentrations. Although FTY720 treatment significantly decreased the immunoreactivity of IgA, no significant difference was observed in the number of duodenal $\mathrm{IgA}^{+}$cells. Considering that there was a time lag in the decrease of fecal and serum IgA concentration upon FTY720 treatment, a significant decrease in the number of duodenal $\operatorname{Ig} \mathrm{A}^{+}$cells may have been detected with a longer observation period. In contrast to IgA, the expression of IgG in the feces, sera, and intestines was not altered by FTY720 treatment. These results suggest that FTY720 specifically blocked the intestinal IgA production in dogs, which is consistent with the results of a previous study in mice [20]. There are a few potential mechanisms for the FTY720 selectivity of IgA: FTY720 may specifically inhibit the migration of $\operatorname{IgA}^{+}$B cells into the intestines, or FTY720 may directly downregulate the IgA production. Further in vitro studies will be necessary to evaluate the direct effect of FTY720 on the immunoglobulin production.

Interestingly, the intestinal S1P concentration in dogs with IBD was significantly lower than that in healthy dogs. Furthermore, the expression levels of S1P1 were significantly decreased in dogs with IBD compared with healthy dogs, indicating that the S1P/ S1P1 axis is attenuated in the intestine of dogs with IBD used in this study. S1P is produced by the activation of synthetic enzymes SK1 and SK2, whereas it is degraded by the S1P lyase, SGPL1 [29, 32, 34]. This study showed that the SK1 mRNA expression was significantly decreased in the duodenal mucosa of dogs with IBD, whereas that of SK2 and SGPL1 was not. These results suggest that the decreased SK1 expression in dogs with IBD is involved in the impairment of S1P production. SK1 expression was induced by estrogen [35], EGF [8], and vitamin D [25]. It has been reported that hypovitaminosis D is associated with gastrointestinal inflammation in dogs with IBD $[1,37]$. Thus, we hypothesize that the low SK1 expression in dogs with IBD may be associated with hypovitaminosis D. Further studies would be necessary to evaluate the relationship between vitamin D and SK1 expression.

This study has several limitations. First, the evaluation of circulating lymphocyte subsets was lacking. Second, the changes in intestinal lymphocytes and gene expression levels were evaluated only in the duodenum. Third, we did not investigate the protein levels of S1P-related molecules, because of the lack of antibodies for these molecules. Further investigations based on IHC or ELISA, using mucosal samples from different parts of intestinal tract, would be needed for a more comprehensive understanding of the topic.

In conclusion, this study showed that the S1P signal plays an important role in lymphocyte trafficking, especially that of B cells, as well as intestinal IgA production. Moreover, we detected a decreased production of intestinal S1P, likely caused by a lower SK1 expression, in a subset of dogs with IBD. This study showed one possible pathophysiological mechanism for canine IBD. However, the present results do not allow us to conclude whether the decreased S1P plays a role in the pathogenesis of IBD. Additional research would be necessary to investigate the importance of the S1P signal and intestinal IgA in the mucosal immunity.

ACKNOWLEDGMENTS. This study was supported by JSPS KAKENHI Grant-in-Aid for Young Scientists (A) (grant no. JP16H06208) and Anicom Capital Research Grant (EVOLVE).

\section{REFERENCES}

1. Allenspach, K., Rizzo, J., Jergens, A. E. and Chang, Y. M. 2017. Hypovitaminosis D is associated with negative outcome in dogs with protein losing enteropathy: a retrospective study of 43 cases. BMC Vet. Res. 13: 96. [Medline] [CrossRef]

2. Aoki, M., Aoki, H., Ramanathan, R., Hait, N. C. and Takabe, K. 2016. Sphingosine-1-phosphate signaling in immune cells and inflammation: roles and therapeutic potential. Mediators Inflamm. 2016: 8606878. [Medline]

3. Blaho, V. A. and Hla, T. 2014. An update on the biology of sphingosine 1-phosphate receptors. J. Lipid Res. 55: 1596-1608. [Medline] [CrossRef]

4. Burgener, I. A., König, A., Allenspach, K., Sauter, S. N., Boisclair, J., Doherr, M. G. and Jungi, T. W. 2008. Upregulation of toll-like receptors in chronic enteropathies in dogs. J. Vet. Intern. Med. 22: 553-560. [Medline] [CrossRef]

5. Cerutti, A. and Rescigno, M. 2008. The biology of intestinal immunoglobulin A responses. Immunity 28: 740-750. [Medline] [CrossRef]

6. Chiba, K. 2005. FTY720, a new class of immunomodulator, inhibits lymphocyte egress from secondary lymphoid tissues and thymus by agonistic activity at sphingosine 1-phosphate receptors. Pharmacol. Ther. 108: 308-319. [Medline] [CrossRef]

7. Cyster, J. G. 2005. Chemokines, sphingosine-1-phosphate, and cell migration in secondary lymphoid organs. Annu. Rev. Immunol. 23: $127-159$. [Medline] [CrossRef]

8. Döll, F., Pfeilschifter, J. and Huwiler, A. 2005. The epidermal growth factor stimulates sphingosine kinase-1 expression and activity in the human mammary carcinoma cell line MCF7. Biochim. Biophys. Acta 1738: 72-81. [Medline] [CrossRef]

9. German, A. J., Hall, E. J. and Day, M. J. 2001. Immune cell populations within the duodenal mucosa of dogs with enteropathies. J. Vet. Intern. Med. 15: 14-25. [Medline] [CrossRef]

10. German, A. J., Hall, E. J. and Day, M. J. 2003. Chronic intestinal inflammation and intestinal disease in dogs. J. Vet. Intern. Med. 17: 8-20. [Medline] [CrossRef]

11. Gohda, M., Kunisawa, J., Miura, F., Kagiyama, Y., Kurashima, Y., Higuchi, M., Ishikawa, I., Ogahara, I. and Kiyono, H. 2008. Sphingosine 1-phosphate regulates the egress of IgA plasmablasts from Peyer's patches for intestinal IgA responses. J. Immunol. 180: 5335-5343. [Medline] [CrossRef] 
12. Goto-Koshino, Y., Ohno, K., Nakajima, M., Mochizuki, H., Kanemoto, H. and Tsujimoto, H. 2011. A rapid and simple method to obtain canine peripheral blood-derived macrophages. J. Vet. Med. Sci. 73: 773-778. [Medline] [CrossRef]

13. Gräler, M. H. and Goetzl, E. J. 2004. The immunosuppressant FTY720 down-regulates sphingosine 1-phosphate G-protein-coupled receptors. FASEB J. 18: 551-553. [Medline] [CrossRef]

14. Gutzeit, C., Magri, G. and Cerutti, A. 2014. Intestinal IgA production and its role in host-microbe interaction. Immunol. Rev. 260: 76-85. [Medline] [CrossRef]

15. Halin, C., Scimone, M. L., Bonasio, R., Gauguet, J. M., Mempel, T. R., Quackenbush, E., Proia, R. L., Mandala, S. and von Andrian, U. H. 2005. The S1P-analog FTY720 differentially modulates T-cell homing via HEV: T-cell-expressed S1P1 amplifies integrin activation in peripheral lymph nodes but not in Peyer patches. Blood 106: 1314-1322. [Medline] [CrossRef]

16. Honneffer, J. B., Minamoto, Y. and Suchodolski, J. S. 2014. Microbiota alterations in acute and chronic gastrointestinal inflammation of cats and dogs. World J. Gastroenterol. 20: 16489-16497. [Medline] [CrossRef]

17. Jergens, A. E., Moore, F. M., Haynes, J. S. and Miles, K. G. 1992. Idiopathic inflammatory bowel disease in dogs and cats: 84 cases (1987-1990). J. Am. Vet. Med. Assoc. 201: 1603-1608. [Medline]

18. Jergens, A. E., Sonea, I. M., O’Connor, A. M., Kauffman, L. K., Grozdanic, S. D., Ackermann, M. R. and Evans, R. B. 2009. Intestinal cytokine mRNA expression in canine inflammatory bowel disease: a meta-analysis with critical appraisal. Comp. Med. 59: 153-162. [Medline]

19. Kimura, T., Boehmler, A. M., Seitz, G., Kuçi, S., Wiesner, T., Brinkmann, V., Kanz, L. and Möhle, R. 2004. The sphingosine 1-phosphate receptor agonist FTY720 supports CXCR4-dependent migration and bone marrow homing of human CD34+ progenitor cells. Blood 103: $4478-4486$. [Medline] [CrossRef]

20. Kunisawa, J., Kurashima, Y., Gohda, M., Higuchi, M., Ishikawa, I., Miura, F., Ogahara, I. and Kiyono, H. 2007. Sphingosine 1-phosphate regulates peritoneal B-cell trafficking for subsequent intestinal IgA production. Blood 109: 3749-3756. [Medline] [CrossRef]

21. Macpherson, A. J., McCoy, K. D., Johansen, F. E. and Brandtzaeg, P. 2008. The immune geography of IgA induction and function. Mucosal Immunol. 1: 11-22. [Medline] [CrossRef]

22. Maeda, S., Ohno, K., Fujiwara-Igarashi, A., Uchida, K. and Tsujimoto, H. 2016. Changes in foxp3-positive regulatory T cell number in the intestine of dogs with idiopathic inflammatory bowel disease and intestinal lymphoma. Vet. Pathol. 53: 102-112. [Medline] [CrossRef]

23. Maeda, S., Ohno, K., Uchida, K., Nakashima, K., Fukushima, K., Tsukamoto, A., Nakajima, M., Fujino, Y. and Tsujimoto, H. 2013. Decreased immunoglobulin A concentrations in feces, duodenum, and peripheral blood mononuclear cells of dogs with inflammatory bowel disease. J. Vet. Intern. Med. 27: 47-55. [Medline] [CrossRef]

24. Maeda, S., Ohno, K., Nakamura, K., Uchida, K., Nakashima, K., Fukushima, K., Tsukamoto, A., Goto-Koshino, Y., Fujino, Y. and Tsujimoto, H. 2011. Quantification of chemokine and chemokine receptor gene expression in duodenal mucosa of dogs with inflammatory bowel disease. Vet. Immunol. Immunopathol. 144: 290-298. [Medline] [CrossRef]

25. Manggau, M., Kim, D. S., Ruwisch, L., Vogler, R., Korting, H. C., Schäfer-Korting, M. and Kleuser, B. 2001. 1Alpha,25-dihydroxyvitamin D3 protects human keratinocytes from apoptosis by the formation of sphingosine-1-phosphate. J. Invest. Dermatol. 117: 1241-1249. [Medline] [CrossRef]

26. Matloubian, M., Lo, C. G., Cinamon, G., Lesneski, M. J., Xu, Y., Brinkmann, V., Allende, M. L., Proia, R. L. and Cyster, J. G. 2004. Lymphocyte egress from thymus and peripheral lymphoid organs is dependent on S1P receptor 1. Nature 427: 355-360. [Medline] [CrossRef]

27. McMahon, L. A., House, A. K., Catchpole, B., Elson-Riggins, J., Riddle, A., Smith, K., Werling, D., Burgener, I. A. and Allenspach, K. 2010. Expression of Toll-like receptor 2 in duodenal biopsies from dogs with inflammatory bowel disease is associated with severity of disease. Vet. Immunol. Immunopathol. 135: 158-163. [Medline] [CrossRef]

28. Minamoto, Y., Otoni, C. C., Steelman, S. M., Büyükleblebici, O., Steiner, J. M., Jergens, A. E. and Suchodolski, J. S. 2015. Alteration of the fecal microbiota and serum metabolite profiles in dogs with idiopathic inflammatory bowel disease. Gut Microbes 6: 33-47. [Medline] [CrossRef]

29. Pappu, R., Schwab, S. R., Cornelissen, I., Pereira, J. P., Regard, J. B., Xu, Y., Camerer, E., Zheng, Y. W., Huang, Y., Cyster, J. G. and Coughlin, S. R. 2007. Promotion of lymphocyte egress into blood and lymph by distinct sources of sphingosine-1-phosphate. Science 316: 295-298. [Medline] [CrossRef]

30. Peyrin-Biroulet, L., Christopher, R., Behan, D. and Lassen, C. 2017. Modulation of sphingosine-1-phosphate in inflammatory bowel disease. Autoimmun. Rev. 16: 495-503. [Medline] [CrossRef]

31. Pham, T. H. M., Okada, T., Matloubian, M., Lo, C. G. and Cyster, J. G. 2008. S1P1 receptor signaling overrides retention mediated by G alpha i-coupled receptors to promote T cell egress. Immunity 28: 122-133. [Medline] [CrossRef]

32. Pyne, S., Adams, D. R. and Pyne, N. J. 2016. Sphingosine 1-phosphate and sphingosine kinases in health and disease: Recent advances. Prog. Lipid Res. 62: 93-106. [Medline] [CrossRef]

33. Rodriguez, A. M., Graef, A. J., LeVine, D. N., Cohen, I. R., Modiano, J. F. and Kim, J. H. 2015. Association of sphingosine-1-phosphate (S1P)/S1P receptor-1 pathway with cell proliferation and survival in canine hemangiosarcoma. J. Vet. Intern. Med. 29: 1088-1097. [Medline] [CrossRef]

34. Spiegel, S. and Milstien, S. 2011. The outs and the ins of sphingosine-1-phosphate in immunity. Nat. Rev. Immunol. 11: 403-415. [Medline] [CrossRef]

35. Sukocheva, O. A., Wang, L., Albanese, N., Pitson, S. M., Vadas, M. A. and Xia, P. 2003. Sphingosine kinase transmits estrogen signaling in human breast cancer cells. Mol. Endocrinol. 17: 2002-2012. [Medline] [CrossRef]

36. Suzuki, T., Jin, M. B., Shimamura, T., Yamashita, K., Taniguchi, M., Nomura, M., Yokota, R., Fukai, M., Magata, S., Horiuchi, H., Fujita, M., Nagashima, K., Furukawa, H. and Todo, S. 2004. A new immunosuppressant, FTY720, in canine kidney transplantation: effect of single-drug, induction and combination treatments. Transpl. Int. 17: 574-584. [Medline] [CrossRef]

37. Titmarsh, H. F., Gow, A. G., Kilpatrick, S., Cartwright, J. A., Milne, E. M., Philbey, A. W., Berry, J., Handel, I. and Mellanby, R. J. 2015. Low vitamin D status is associated with systemic and gastrointestinal inflammation in dogs with a chronic enteropathy. PLoS One 10: e0137377. [Medline] [CrossRef]

38. Yagi, H., Kamba, R., Chiba, K., Soga, H., Yaguchi, K., Nakamura, M. and Itoh, T. 2000. Immunosuppressant FTY720 inhibits thymocyte emigration. Eur. J. Immunol. 30: 1435-1444. [Medline] [CrossRef]

39. Yopp, A. C., Fu, S., Honig, S. M., Randolph, G. J., Ding, Y., Krieger, N. R. and Bromberg, J. S. 2004. FTY720-enhanced T cell homing is dependent on CCR2, CCR5, CCR7, and CXCR4: evidence for distinct chemokine compartments. J. Immunol. 173: 855-865. [Medline] [CrossRef] 